\title{
Online Monitoring for the CDF Run II Experiment and the Remote Operation Facilities
}

T.Arisawa $^{a}$, D.Fabiani $^{b}$, D.Hirschbuehl $^{c}$, K.Ikado $^{a}$, T.Kubo $^{d}$, Y.Kusakabe $^{a}$, K.Maeshima $^{e}$, J.Naganoma ${ }^{a}$, K.Nakamura ${ }^{a}$, C.Plager ${ }^{e}$, E.Schmidt $^{f}$, F.Scuri $^{b *_{;}}$, H.Stadie ${ }^{c}$, R.Tsychiya ${ }^{a}$, G.Veramendi ${ }^{g}$, W.Wagner ${ }^{c}$, H.Wenzel ${ }^{c}$, M.Worcester ${ }^{f}$, K. Yorita ${ }^{h}$

${ }^{a}$ Waseda University, Tokyo, Japan

${ }^{b}$ Istituto Nazionale di Fisica Nucleare, Pisa, Italy

${ }^{c}$ Universitaet Karlsruhe, Germany

${ }^{d}$ K.E.K, Tsukuba, Japan

${ }^{e}$ University of California at Los Angeles, U.S.

${ }^{f}$ Fermilab, Batavia (IL), U.S.

${ }^{g}$ L.B.L., Berkeley (CA), U.S.

${ }^{h}$ University of Chicago, U.S.

The foundation of the CDF Run II online event monitoring framework, placed well before the physics runs start, allowed to develop coherent monitoring software across all the different subsystems which consequently made maintenance and operation simple and efficient. Only one shift person is needed to monitor the entire CDF detector, including the trigger system. High data quality check is assured in real time and well defined monitoring results are propagated coherently to offline datasets used for physics analyzes. We describe the CDF Run II online event monitoring system and operation, with enphasis on the remote monitoring shift operation started since November 2006 with Pisa-INFN as pilot Institution and exploiting the WEB based access to the data.

XI International Workshop on Advanced Computing and Analysis Techniques in Physics Research April 23-27 2007

Amsterdam, the Netherlands

\footnotetext{
* Speaker.

${ }^{\dagger}$ E-mail: fabrizio.scuri@pi.infn.it
} 


\section{Introduction}

The CDF experiment is data taking since 2002 at the Run-II Fermilab Tevatron collider operating proton-antiproton interactions at $1.96 \mathrm{TeV}$ center of mass energy. The $\mathrm{CDF}$ detector, whose detailed description can be found elsewhere [1], has a typical architecture for high energy experiments, divided into a tenth of subdetectors including: inner tracking systems and Time of Flight detetctors, central superconducting solenoid $(1.4 \mathrm{~T})$, outer calorimeters and muon detectors, 3-level trigger system, multi-step data flow chain (250 dual processors for L3 trigger, robotic tape storage, 150 dual processors for the production farm, 9 Distributed Central Analysis Facilities), and a 20 PC cluster for the online operations. The online structures include the Data Aquisition system, monitors for detector calibration and data taking operation, event display, and Web servers. The CDF detector and data flow complexity are similar to those of the experiments designed for the LHC program; in particular, the following specs summarize the CDF operation highlights:

- 0.75 Million channels to be operated and monitored.

- $75 \mathrm{~Hz}$ output of the Level 3 trigger at $20 \mathrm{MB} / \mathrm{s}$ (system limit), corresponding to about 0.5 $\mathrm{PB} /$ year of data throughput.

The main characteristics of the architecture of the CDF online system are described in section 2; some details of the new CDF remote monitoring operations are given in section 3; conclusions are summarized in section 4 .

\section{Architecture of the CDF online system}

The main characteristics of the CDF online system are described in this section; a more detailed description can be found elsewhere [2]. The online architecture was designed to match the following requirements:

- DAQ, Trigger, and detector performances must be monitored without interfering with the data taking.

- Monitored results must be interpreted by the crew on shift in a fast and clear way in order to mantain high quality data taking.

- Different monitor (Consumer) processes can run on different machine to allow the system to be easily expanded.

- each Consumer receives only the data it needs according to the trigger selections.

- Monitor and Display process types are separated; the number of active displays are limited by the network traffic and the bandwidth.

- Different Consumer processes can be combined to one executable.

- Online software is developped within the CDF sofware environment as a standard detector partition to simplify maintainability. 
- Save remote access to the monitoring operations and maintainance must be grant to the remote shifters and to the experts.

The raw scheme of the CDF online monitoring architecture is shown in figure 1.

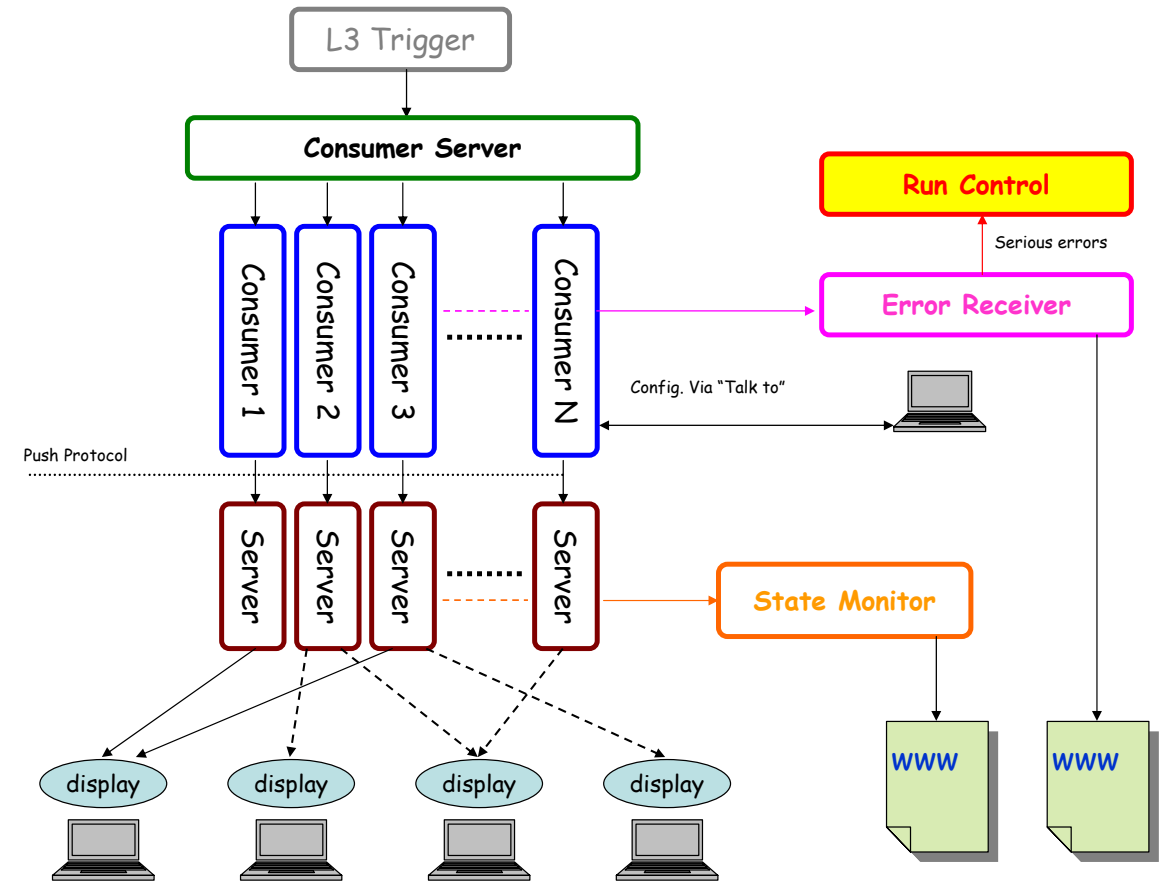

Figure 1: Raw architecture of the CDF online monitoring

Data relevant to the monitor operation are produced by the Consumers, receiving the input from the Consumer servers, continuosly running during data aquisition, and updating the Display Servers. Display operations are operator driven via Display Browsers which are programs passing to the user endnode the data received from the Display Servers; Display Browsers can run on the same machine as the Display Servers or on different machines either via a socket connection or via WEB through a plug-in for the APACHE [3] webserver to maintain ROOT [4] compatibility (figure 2).

Examples of monitor displays are shown in figure 3. All monitor displays are updated each second; they are very useful to promptly detect new dead regions (contiguous white cells in the example shown in figure 3). Histograms produced by the displays are available on WEB after update requests and, very recently, snapshot pictures of all DAQ and online screens in the CDF control room were let available on WEB (down to $5 \mathrm{sec}$. update) for remote monitoring porposes.

All Consumer and Monitor programs are based on $\mathrm{C}++$ modules of Analisys Control++ (AC++) [5]; AC++ is the CDF analysis framework handling dataflow between modules; it serves input, output, event generation, detector simulation, event reconstruction, access to the database, and physics analyses; ROOT is the SW package used for event I/O and analysis tools; several version of the CDF SW package are released each year and the online modules must follow the SW evolution; each specific consumer/monitor is written according to the sub-detector specs, within the CDF SW 


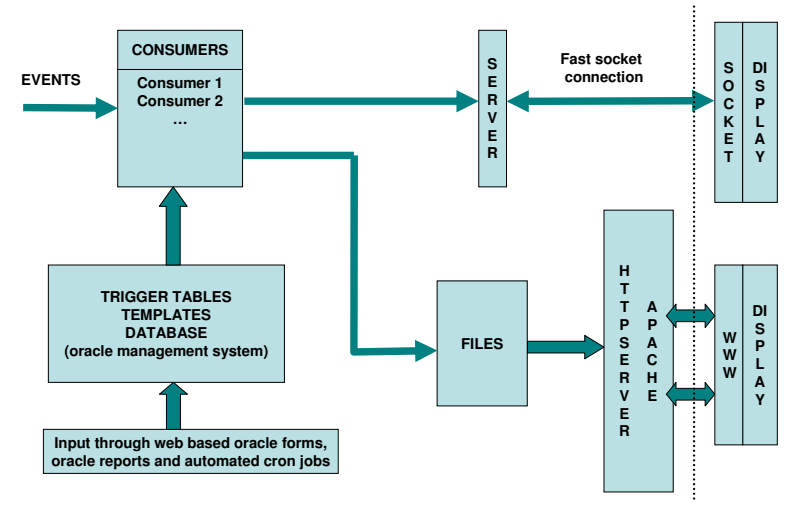

Figure 2: Element communication layout

framework; template programs are used with utility methods to access data and to produce histograms without interfering with the data acquisition; standard procedures are defined to compare actual and reference plots and to provide instructions to the operators.

Typical monitored objects are: detector occupancies, trigger rates, luminosity, vertex position, physics objects, L3 reconstruction, and calibration results.

Just one person in shift (Consumer Operator) can handle full detector and data flow monitoring; sub-detector experts are paged only in case of major troubles.

\section{Remote monitoring operations}

The main job for the Consumer Operator (CO) is to online check the detector operation and data quality by fulfilling the following duties:

a) by looking at the:

- Consumer Monitor outputs;

- Detector Calibration results;

- Event Display.

b) by submitting the result of a detailed check-list of the monitored objects.

Once grant the possibility to access from remote to the full information and to the operations requested to serve the $\mathrm{CO}$ shift, there is no reason for the $\mathrm{CO}$ operator to be physically in the $\mathrm{CDF}$ control room. Therefore, CDF decided to move from local to remote all owl CO shifts. This has a strong impact for the members of the CDF collaboration of European and Asian Institutions and for the CDF shift organization itself. Exploiting the regional time difference, owl CDF CO shifts can be assigned to European or Asian members, making remote shifts in a more confortable day time and, at the same time, reducing CDF the number of people on night shift to the Scientific Coordinator and DAQ operator.

Tsukuba (KEK, Japan) and Pisa (Italy) were chosen as pilot Institutions for the CDF Remote $\mathrm{CO}$ shift project; the project developed in cooperation with CMS-Fnal group, which is setting-up 


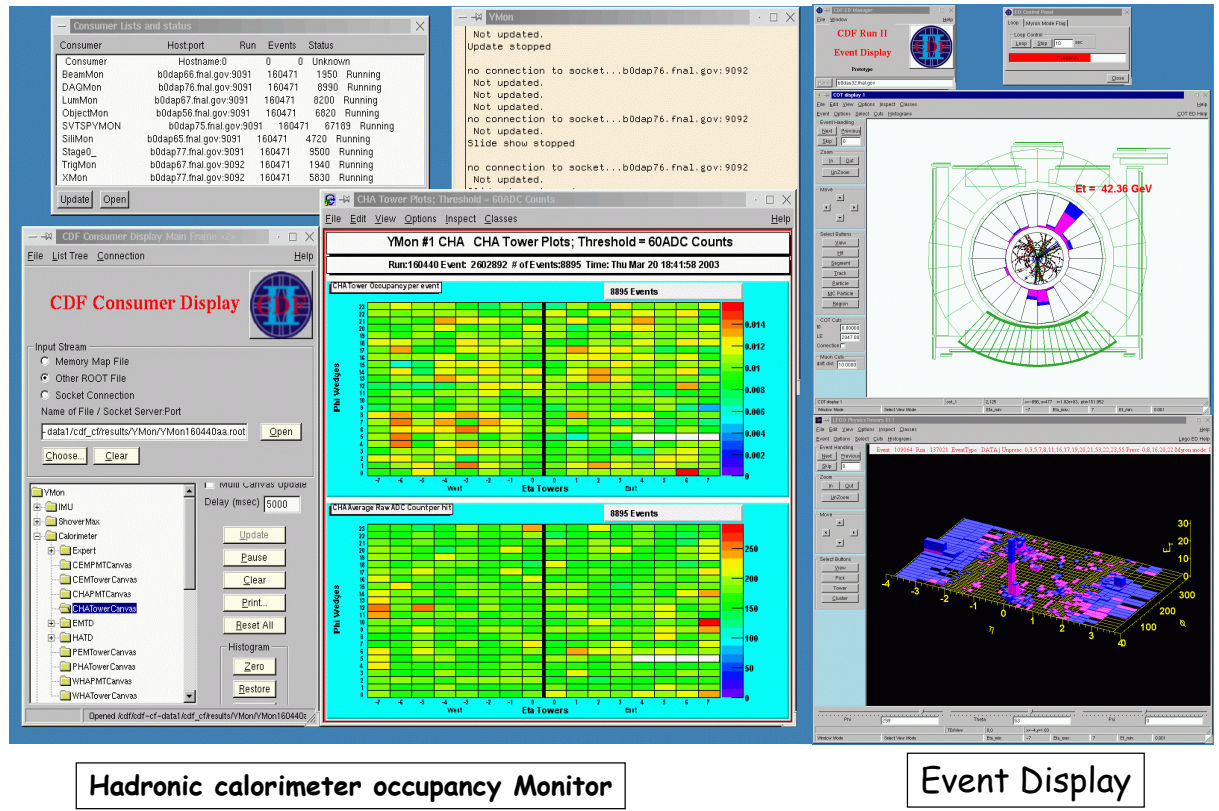

Figure 3: Examples of monitor displays.

a multipurpose remote control room in the Wilson Hall at Fnal, and with the aim to acquire the know-how for future applications of remote shifts (LHC, ILC).

The remote shift project pushed the online group to make all the upgrades required to allow full remote operation; this resulted in a general improvement and simplification of the Consumer Operator shift; among several upgrades made to allow all quality and status checks via WEB, we just mention one relative to the $\mathrm{CO}$ specific operations during the shift, i.e. the possibility to remotely change the run mode (Physics/Comsmic) and to start/restart Consumers and Displays. These operations are normally made in local by running command scripts from any machine of the online cluster. However, remote login on the online cluster from outside Fnal is forbidden by the Fnal-D.o.E. policy for people other than online experts. To overcome this limitation and allow the remote shifter to operate the executables, a new GUI with action bottons was developed; the GUI window is exported from Fnal to the authorized remote node with a simple script launched by the local crew once at the beginning of the remote shift. The new GUI, named ConCon (Consumer Control), is based on ROOT classes and merges in a user friendly way all action alternatively performed in local with a lot of shell commands launching script files. The ConCon GUI window is described in figure 4 .

Commissioning of the remote shift procedure and first standard remote shifts were done from Pisa; the initial set-up of the remote control room is quite simple; a desktop equipped with a webcam and running Polycom PVX under Windows XP was devoted to the video-connection between 


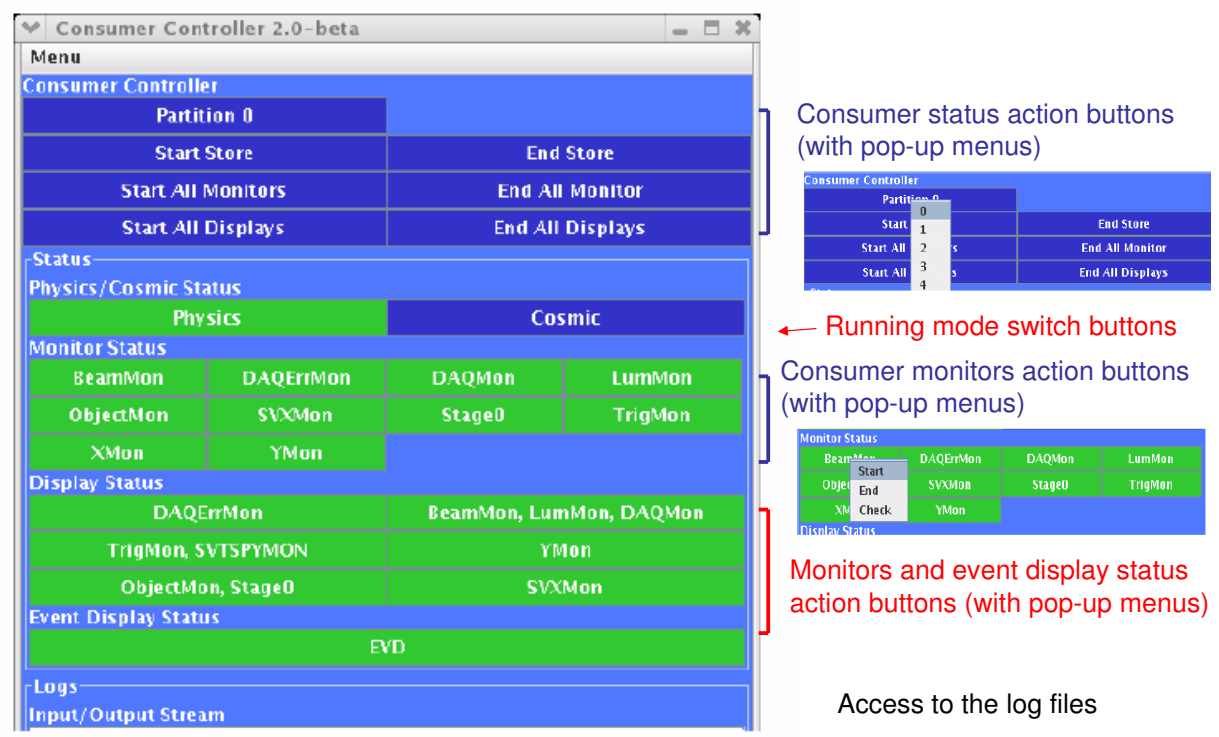

Figure 4: The ConCon GUI window.

local and remote control rooms; a second desktop with a four-screen drive card was used for the normal shift operation, normally histogram checking from WEB browsers, running under Scientific Linux. The remote system will be upgraded with a second desktop driving 6 additional monitors where real time updated snapshots of the DAQ, slow control, and online screens in the local (Fnal) control room will be observed. Figure 5 shows the local (Fnal) section of the CDF control room devoted to the Consumer Operator monitoring (top-left) and the CDF remote control room in Pisa (botom-right).

Commissioning of the remote shift procedures was completed in the period September-November 2006; in this period the details of the start-up procedure for the remote shifts in Pisa and for emergency situation handling were defined, e.g. remote shifter replacement with a local (Fnal) one in case of any failure at the start-up and in case of network or HW failures during the shift. New HW (Pisa and Fnal) for video-connection and monitoring (Pisa) was tested as well the new SW for remote shift (new control GUI and new WEB based procedures).

Pilot remote CO shifts were made in November 2006: two 8 day regular owl shifts, Pisa and Fnal in overlap were performed to check the long term network connection reliability, which was considered the main issue (short network breakdowns ( $\leq 15 \mathrm{~min}$.) are not an issue).

Regular remote CO shift operation started on December 2006: at the beginning 4 shift (one per month) were done until March 2007, then 2 shifts per month were scheduled from April 2007; $6 \times 8$ day shift statistics is very encouraging: no HW, nor SW problems detected, no network 


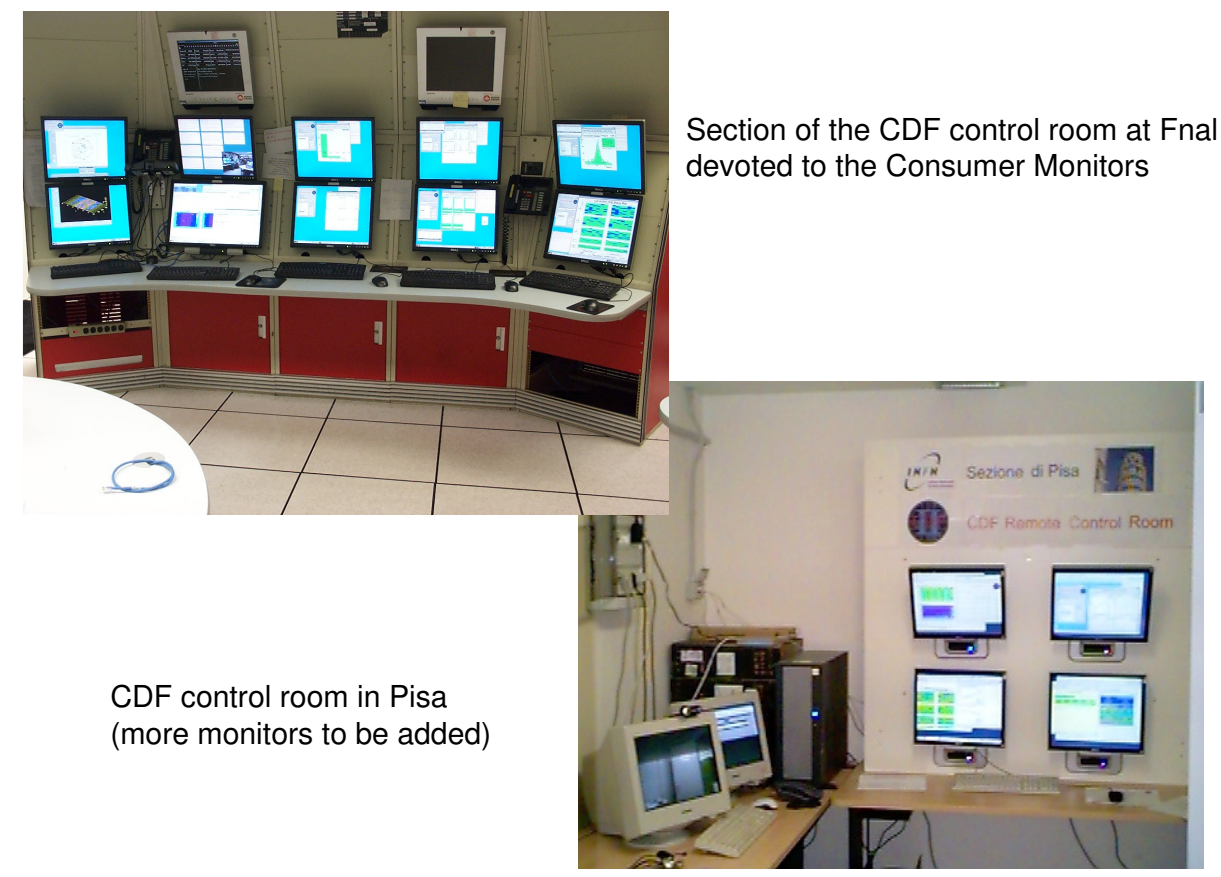

Figure 5: The CDF local (top-left) and remote (bottom-right) control rooms

connectivity breakdowns, just $15 \mathrm{~min}$. interruption due to a scheduled maintenance.

\section{Conclusions}

The CDF online system is reliably operating since several years; the system architecture and SW upgrades smoothly developed according to the CDF SW environment evolution.

Real time monitoring of the detector status and of data quality is now fully operated also from remote both for expert feedbacks and for quality checking and monitor (Consumer Operators shifts); local and remote CO shifts proceed now exactly in the same way.

Changes required for the remote shifts resulted in a global improvement towards a more user friendly environment.

First period operation of remote shifts from Pisa is very positive; based on the Pisa experience, CDF plans to operate other remote sites and to achieve assignment to remote $100 \%$ owl CO shifts. We encourage other worldwide experiment communities to follow this example.

\section{References}

[1] R. Blair at al. (CDF Collaboration), "The CDF II Detector - Technical Design Report", FERMILAB-PUB-96-390-E (1996) 
[2] T. Arisawa et al.,"Online Monitoring for the CDF Run II Experiment", proceeding of CHEP 2003, March 24-28 2003, La Jolla, California

W. Wagner et al., "Online Monitoring in the CDF II Eperiment", proceedings of HEP2001, 12-18 July 2001, Budapest, Hungary, hep2001/273.

[3] The Apache Software Foundation, http://www.apache.org

[4] R. Brun and F. Rademakers, Nucl.Instrum.Meth. A389:81-86,1997.

[5] E. Sexton-Kennedy," A Users Guide to the AC++ Framework", CDF public note, CDF/DOC/CDF/COMP_UPG/PUBLIC/4178 